\title{
Comparison and clinical implementation of quality of life tools in patients with small bowel neuroendocrine tumors treated with LU-DOTA-TATE PRRT
}

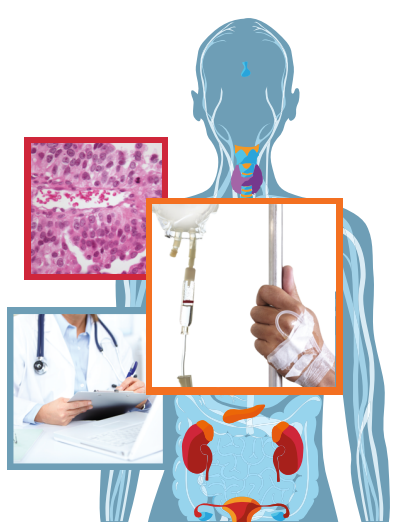

International Journal of Endocrine Oncology

\author{
Bianka Saravana-Bawan¹, Stella Koumna², Marguerite Wieler ${ }^{3}$, Alexander McEwan² \& Todd \\ McMullen*,1 \\ ${ }^{1}$ Department of Surgery, University of Alberta, Edmonton, AB, T6G 2R3, Canada \\ ${ }^{2}$ Department of Oncology, University of Alberta, Edmonton, AB, T6G 2R3, Canada \\ ${ }^{3}$ Department of Physical Therapy, University of Alberta, Edmonton, AB, T6G 2R3, Canada \\ *Author for correspondence: Tel.: +1 780407 1108; Fax: +1 780407 2729; tpm1@ualberta.ca
}

\begin{abstract}
Aim: This study assesses if clinically developed quality of life (QoL) tools are as effective in small bowel neuroendocrine tumors (NETs) as NET-specific research questionnaires. Methods: QoL in patients with small bowel NETs treated with Lu-DOTA-TATE was assessed with The European Organization for Research and Treatment of Cancer (EORTC) QLQ-C30, QLQ-GI.NET21 and Edmonton Symptom Assessment System Revised (ESAS-r) at baseline and after four treatments. Repeated measures ANOVA was performed. Results: Both EORTC and ESAS-r demonstrated maintained overall QoL. EORTC demonstrated statistically and clinically significant improvement in insomnia, diarrhea, gastrointestinal, endocrine symptoms and social function. ESAS-r demonstrated statistically and clinically significant improvement in overall total symptom distress score. Conclusion: ESAS-r is quick and easy to interpret. It is not as sensitive to individual symptoms but does track overall function. EORTC assessment is more complex, but better reflects QoL for NET specific symptoms.
\end{abstract}

First draft submitted: 26 April 2019; Accepted for publication: 16 August 2019; Published online: 19 September 2019

Keywords: lutetium • NET • neuroendocrine tumor $\bullet$ PRRT $\bullet$ QoL $\bullet$ quality of life

There has been a conscious shift in the practice of medicine from a disease-centered model of care to one which is patient directed [1]. To facilitate this patient directed care, there is a need for better integration of the patient perspective $[2,3]$. Empowering patients to discuss factors not limited to direct disease-related symptoms, but also to subjective parameters such as social and psychological elements, improves outcomes. Patient self-reports of health status in systematic assessments vary significantly from what is volunteered in clinical assessments, with the former eliciting more extensive symptomology [4,5]. Physician assessment often focuses on objective measures such as imaging and does not necessarily accurately represent the patient experience [6].

Consequently, the evaluation of patient-reported quality of life $(\mathrm{QoL})$ through validated questionnaires have been increasingly included in clinical practice guidelines $[7,8]$. These measures are especially important in the care of cancer patients with advanced disease in which treatment is not of curative intent, but to minimize symptoms and maintain lifestyle $[4,9,10]$. The National Comprehensive Cancer Network recognizes the disease-specific importance for management of multiple patient domains and recommends that all patients be screened for QoL through the course of their management [11].

Patients with neuroendocrine tumors (NETs) often survive 5 or 10 years with progressive metastatic disease. As the majority of grade 1 and grade 2 gastroenteropancreatic (GEP)-NETs are slow growing, even patients with metastatic disease have a long life expectancy [12-15]. A significant proportion of all patients with GEP-NETs will ultimately present with a wide variety of symptoms that may be systemic (i.e., flushing), as well as organ-specific (i.e., small bowel) [14-18]. Symptoms with NETs can be related to tumor burden or tumor hormonal secretion. NETs can secrete a variety of hormones including serotonin, somatostatin, gastrin, insulin and histamine [19]. Of the

Future Medicine 
functional NETs, serotonin secretion is the most common and up to $20 \%$ of patients develop carcinoid syndrome, which is characterized by flushing, diarrhea, abdominal pain, bronchoconstriction and potentially carcinoid heart disease [19]. Nonsecretory tumors do not secrete hormones. In these patients, symptoms are largely related to tumor burden and disease progression. Symptoms of locoregionally advanced and metastatic GEP-NETs can include abdominal pain, hepatobiliary obstruction and bowel obstruction. Nonadvanced small bowel NETs (SB-NET) can also exhibit symptoms of bowel obstruction secondary to the associated mesenteric fibrotic reaction exhibited by NETs. Consequently, for these patients, questionnaires assessing QoL should include general as well as NET-specific domains [20,21].

Studies have demonstrated an improvement in QoL parameters in patients with NETs treated with Lu-DOTATATE including but not limited to improvement in global health status, physical functioning and pain [22-25]. Most recently and notable NETTER-1 trial updated results have demonstrated improved time to deterioration in multiple QoL parameters including global health status, physical functioning and pain in patients treated with Lu-DOTA-TATE and octreotide long-acting repeatable in comparison to octreotide long-acting repeatable alone [26].

However, the use of QoL assessment tools in clinical cancer care practice is still somewhat limited [27]. While QoL assessment has been utilized, the research setting uptake in clinical practice has not followed. This uptake of QoL assessment in routine clinical care can assist in improving patient outcomes by providing direct and quantifiable feedback that clinicians and patients can monitor and use to modify treatment plans.

A main limiting factor in adopting patient-directed symptom tools into clinical practice is the time requirement and learning curve regarding administration, scoring and interpretation of results [9]. Assessment tools are often designed for research and can be difficult for clinicians to understand with regards to results and scoring systems. Ideal characteristics of a QoL questionnaire for utilization in the clinical domain, rather than research environment, are that they are brief, easy for the patient to complete without assistance and easy for clinician to score and interpret [28,29].

The European Organization for Research and Treatment of Cancer (EORTC) Quality of Life QuestionnaireCore 30 (QLQ-C30) is a validated QoL questionnaire, which assesses different parameters including functional and symptom scales. The QLQ-GI.NET 21 addition adds 21 questions which address NET-specific parameters [30].

Comparatively, the Edmonton Symptom Assessment System Revised (ESAS-r) form is a validated tool which is designed for use in clinical practice. Initially developed by Bruera et al. for palliative care, it is commonly employed in both palliative care and general oncology $[31,32]$. The form can be completed by patients within a few minutes $[4,8,12,13]$. It assesses nine symptoms in addition for an optional tenth category as self reported by the patient [33]. The scoring is simple, requiring no calculations and conversion [33]. Symptoms with a score of 1-3 are clinically considered mild, 4-6 moderate and 7-10 severe.

Consequently, the ESAS-r is a more clinically friendly tool than the EORTC QLQ-C30 with QLQ.GI-NET21 as it does not require physicians to use scoring algorithms and it is easier for patients to complete. As such we hypothesize that the integration of this QoL assessment tool will be more easily accepted and have a higher uptake than the EORTC QLQ-C30. However, as the ESAS-r is not specifically designed for assessment of NETs, it is unclear if it will be able to detect NET-specific symptomology and patient concerns. To date, no studies have evaluated QoL in patients with GEP-NETs using the ESAS-r. In this study, we evaluate the QoL of patients with metastatic GEP-NETs being treated with Lu-DOTA-TATE peptide receptor radiotherapy with both the NETspecific standard, EORTC QLQ-C30 with QLQ.GI-NET21 and ESAS-r to determine if the ESAS-r is able to similarly detect changes in patient QoL. Our goal is to find the tool that is best assesses and detect changes in QoL for patients with GEP-NETs.

\section{Materials \& methods}

Study design

This study was conducted as a part of the Phase II open label clinical trial at the Cross Cancer Institute in Edmonton conducted by the Department of Oncology. The primary study objective is assessment of the efficacy of Lu-DOTATATE therapy for somatostatin receptor positive tumors. Ethics approval were obtained and are maintained with the University of Alberta research ethics board and with Health Canada.

The inclusion criteria of the Phase II trial included age $\geq 14-90$ years of age; presence of a somatostatin receptor positive tumor with at least one tumor site reliably visualized on imaging of at least $1.5 \mathrm{~cm}$ within 26 weeks of enrolment; life expectancy greater than 12 weeks; Eastern Cooperative Oncology Group (ECOG) of $\leq 2$; and 
written informed consent given prior to enrolment. For specific inclusion in this QoL substudy, the scope of patients was limited to those with SB-NETs to allow for a more homogenous patient population. Additionally, age range was limited to patients from 40 to 80 years of age to reduce the influence of age on QoL.

\section{Peptide receptor radiotherapy treatment protocol}

After study enrolment, patients were initially treated with four cycles of induction therapy. Each cycle consisted of pretreatment bloodwork and QoL questionnaires. The questionnaires completed prior to initial therapy served as baseline assessment. Lu-DOTA-TATE therapy, on average $5.55 \mathrm{GBq}$, with a range $2.50-6.11 \mathrm{GBq}$ per protocol depending on patient risk factors for treatment toxicity. Post-treatment bloodwork was then collected at 6 weeks. The subsequent cycle was commenced 2.5-3.5 months post-treatment.

For patients who did not experience progression or toxicity during induction phase, a subsequent maintenance phase was then entered consisting of up to eight cycles, for a total of 12 potential treatments. Maintenance LuDOTA-TATE therapy dosing was on average $2.78 \mathrm{GBq}$, with a range of $1.67-4.07 \mathrm{GBq}$ varying again in relation to patient risk factors for treatment toxicity. Maintenance cycles consisted of identical bloodwork and QoL assessments in comparison with induction cycles; however, cycles were spaced less frequency at every 6 months.

\section{QOL evaluation}

QoL assessments during treatment used the EORTC QLQ-C30 Version 3.0 (includes QLQ-GI.NET 21) and the ESAS-r tool. Since study commenced in March 2014, all patients have completed the EORTC QLQ-C30. The QLQ-GI.NET 21 and ESAS-r were approved for and introduced into study protocol and subsequently completed by patients as of May 2014.

All questionnaires were administered at the same time and were completed prior to each treatment. After completion of treatment, all questionnaires were also administered at 6 month and 1 year follow-up visits. Baseline QoL was considered to be that established by the questionnaires completed prior to initial Lu-DOTA-TATE treatment. All questionnaires were completed in a paper-based format.

The QLQ-C30 is a validated general QoL tool with the QLQ-GI.NET 21 being validated for the assessment of patients with SB-NETs. The QLQ-C30 contains 30 questions which assess global health status, symptom scales (fatigue, nausea and vomiting, pain, dyspnea, insomnia, appetite loss, constipation, diarrhea and financial difficulties) and functional scales (physical functioning, role functioning, emotional functioning, cognitive functioning and social functioning). The QLQ-GI.NET21 consists of the addition of 21 questions to the QLQ-C30, which assess NET-specific parameters including endocrine symptoms, gastrointestinal symptoms, treatment-related symptoms and disease-related worries. All parameters are evaluated using single or multi-item questions which are consequently transformed through a set protocol to 100 point scores. For global health status and functional scales within the EORTC scale higher scores relate to improved QoL, whereas for the symptom scales lower scores indicate improved QoL [30,34]. Clinically significant changes in QoL as measured by the EORTC QLQ-C30 was considered to be a mean score change of 5-10 points for improvement as established by Osoba et al. in 1998 [35-37].

The ESAS-r consists of nine single item parameters (pain, fatigue, drowsiness, nausea, appetite, dyspnea, depression, anxiety and wellbeing). Each parameter is assessed by the patient on a numerical rating scale from 0 to 10 with 0 indicating no symptoms and 10 indicating the most severe symptom intensity [32,38]. A score of seven indicates severe symptomology [27]. There is also a final single line item allowing for the patient to provide and rate their own area of concern. The overall scores can also be calculated consisting of the physical symptom, emotional symptom and total symptom distress score. The total symptom distress score has a maximum score of 90 and is the sum of all nine parameter single item scores. The physical symptom score has a maximum score of 60 and consists of the sum of the six physical parameters (pain, fatigue, drowsiness, nausea, appetite and dyspnea). The emotional symptom score has a maximum score of 20 and consists of the sum of the two emotional parameters (anxiety and depression) [32,38]. The ESAS-r is a validated QoL tool and has been validated for use in patients with advanced cancer [39]. Clinically significant changes in QoL by the ESAS-r were first determined by Hui et al. in 2015 with optimal cutoff established at a difference of one point for both improvement and deterioration for single scores [40]. For physical and total symptom distress scores, the minimum clinically important difference (MCID) for improvement is referenced in the literature at a decrease in score of three points with the MCID for deterioration being an increase in score of four points. For the emotional symptom distress score the MCID for improvement is established as a score decrease of two points and the MCID for deterioration is a score increase of one point [38]. 


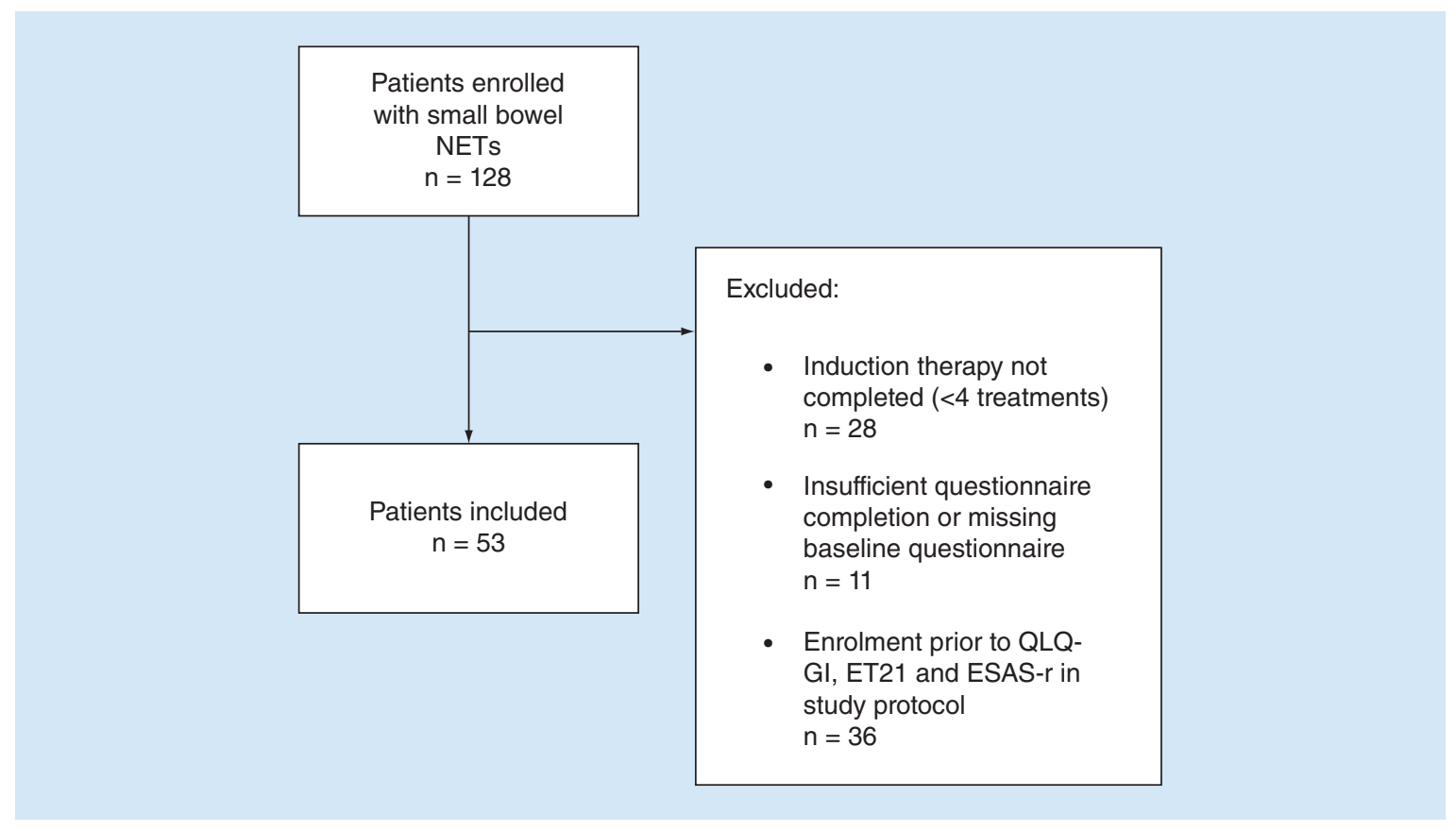

Figure 1. Patient selection for study inclusion. NET: Neuroendocrine tumor.

\section{Data analysis}

QoL assessment was completed during outpatient patient clinics at each pretreatment evaluation. Questionnaire data for all measures were subsequently manually transcribed into the Research Electronic Data Capture interface database established for the trial. Care was taken to carefully instruct patients prior to administration and to provide and collect the questionnaires to minimize missing data. All missing data was treated for the EORTC QLQC-30 and GI.NET 21 as outlined by the EORTC scoring manual guidelines with imputation [30].

Data were analyzed using Stata Version 15 (Stata Corp, TX, USA) and SPSS 21. As planned, interim analysis of induction therapy repeated measure analysis of variance was completed for all patients who had completed the induction therapy consisting of four cycles. All data were initially evaluated to ensure the data met the assumptions of normality and sufficient sample size and equal variance were also confirmed. For repeated measures, testing the assumption of sphericity was also verified prior to data analysis. A p-value of $\leq 0.05$ was considered significant. For EORTC, a threshold of score change of $\geq 5$ was considered to be the MCID [35-37]. For ESAS-r, a threshold of score decrease of $\leq 1$ for individual scores, $\leq 3$ for for physical and total symptom distress score and $\leq 2$ for emotional score was considered to be of MCID.

\section{Results}

\section{Patient baseline characteristics}

A total 53 patients with GI.NETs were eligible for inclusion for QoL analysis as outlined in Figure 1.

The study group included 31 male and 22 female patients with a mean age of 61 years. All patients, with the exception of four, received other treatment prior to Lu-DOTA-TATE treatment (83\% of patients had undergone surgery, $75 \%$ were treated with somatostatin analogs, $9 \%$ had prior chemotherapy, 4\% mammalian target of rapamycin inhibitors or tyrosine kinase inhibitor and $8 \%$ locoregional therapy). All patients had documented disease progression prior to initiation of Lu-DOTA-TATE. Baseline patient characteristics of all patients are further outlined in Table 1 and NET diagnosis of patients are as outlined in Table 2.

QOL

Evaluation of overall QoL as scored by EORTC QLQ-C30 with GI.NET 21 revealed mean global health status of 67.63 at baseline and 65.71 post fourth treatment cycle. Change in global health status from baseline to post-treatment 4 was not statistically significant, $\mathrm{p}=0.72$, and thus demonstrated no improvement but also no 


\section{Table 1. Patient characteristics.}

\begin{tabular}{|c|c|c|}
\hline Patient characteristics & $N(n=53)$ & (\%) \\
\hline \multicolumn{3}{|l|}{ Age: } \\
\hline$-40-49$ & 3 & 6 \\
\hline$-50-59$ & 25 & 47 \\
\hline$-60-79$ & 25 & 47 \\
\hline \multicolumn{3}{|l|}{ Sex: } \\
\hline - Male & 31 & 58 \\
\hline - Female & 22 & 42 \\
\hline \multicolumn{3}{|l|}{ ECOG: } \\
\hline-0 & 35 & 66 \\
\hline-1 & 16 & 30 \\
\hline-2 & 2 & 4 \\
\hline \multicolumn{3}{|l|}{ Previous treatment: } \\
\hline - Surgery & 44 & 83 \\
\hline -SSA & 40 & 75 \\
\hline - Chemotherapy & 5 & 9 \\
\hline - mTOR, TKI & 2 & 4 \\
\hline - Locoregional therapy & 4 & 8 \\
\hline
\end{tabular}

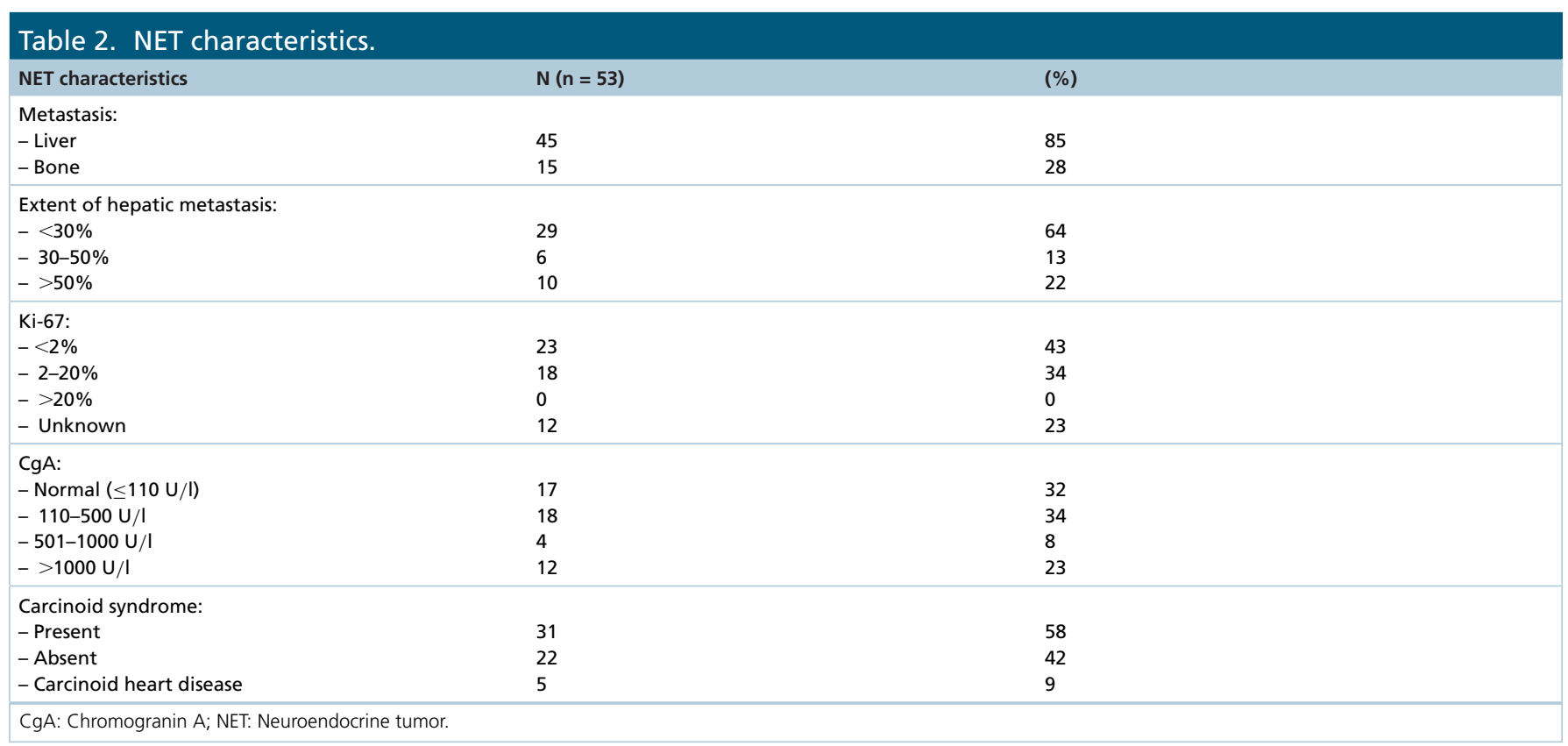

deterioration in overall QoL as assessed by EORTC QLQ-C30 with GI.NET 21. Patient QoL as evaluated by EORTC are outlined in Table 3 and by ESAS-r in Table 4.

Overall QoL scored by ESAS-r revealed mean total symptom distress score of 19.85 at baseline and 15.38 post-treatment four. The change in total symptom distress score was statistically significant, $p=0.01$, indicating in overall QoL as assessed by ESAS-r. Notably, this improvement is also clinically significant with a decrease of 4.47 points meeting the MCID of $\geq 3$ points.

Parameters of QoL as assessed by EORTC QLQ-C30 with GI.NET 21 demonstrated a statistically significant change $(p<0.05)$ in mean scores for insomnia, diarrhea, endocrine symptoms and gastrointestinal (GI) symptoms. All other parameters as detailed in Table 2 were not found to have statistically significant change over the completion of induction treatment.

Mean endocrine symptoms decreased from 21.57 to $15.03(\mathrm{p}=0.04)$, GI symptoms from 24.12 to 15.49 $(\mathrm{p}=0.01)$, diarrhea from 39.74 to $28.21(\mathrm{p}=0.02)$ and insomnia from 36.27 to $24.51(\mathrm{p}=0.04)$. All parameters had a mean changes of $\geq 5$ points indicating a clinically significant change. 
Table 3. European Organization for Research and Treatment of Cancer QLQ C-30 and GI.NET21.

\begin{tabular}{|c|c|c|}
\hline \multirow{2}{*}{ EORTC scale } & \multicolumn{2}{|c|}{ Mean (SD) } \\
\hline & Baseline & Post-treatment 4 \\
\hline Global health status & $\begin{array}{l}67.63 \\
(22.03)\end{array}$ & $\begin{array}{l}65.71 \\
(26.81)\end{array}$ \\
\hline Physical functioning & $\begin{array}{l}77.30 \\
(22.43)\end{array}$ & $\begin{array}{l}80.78 \\
(21.38)\end{array}$ \\
\hline Role functioning & $\begin{array}{l}74.02 \\
(28.78)\end{array}$ & $\begin{array}{l}76.47 \\
(30.46)\end{array}$ \\
\hline Emotional functioning & $\begin{array}{l}69.75 \\
(23.24)\end{array}$ & $\begin{array}{l}72.84 \\
(27.60)\end{array}$ \\
\hline Cognitive functioning & $\begin{array}{l}76.54 \\
(28.84)\end{array}$ & $\begin{array}{l}78.40 \\
(28.43)\end{array}$ \\
\hline Social functioning & $\begin{array}{l}76.54 \\
(27.45)\end{array}$ & $\begin{array}{l}83.95 \\
(25.94)\end{array}$ \\
\hline Pain & $\begin{array}{l}26.96 \\
(29.02)\end{array}$ & $\begin{array}{l}22.06 \\
(30.07)\end{array}$ \\
\hline Dyspnea & $\begin{array}{l}22.55 \\
(31.48)\end{array}$ & $\begin{array}{l}22.55^{*} \\
(25.58)\end{array}$ \\
\hline Insomnia & $\begin{array}{l}36.27 \\
(32.17)\end{array}$ & $\begin{array}{l}24.51^{*} \\
(28.79)\end{array}$ \\
\hline Appetite loss & $\begin{array}{l}14.71 \\
(22.01)\end{array}$ & $\begin{array}{l}10.78 \\
(17.83)\end{array}$ \\
\hline Constipation & $\begin{array}{l}11.76 \\
(24.46)\end{array}$ & $\begin{array}{l}9.80 \\
(26.63)\end{array}$ \\
\hline Diarrhea & $\begin{array}{l}39.74 \\
(28.31)\end{array}$ & $\begin{array}{l}28.21^{*} \\
(29.35)\end{array}$ \\
\hline
\end{tabular}

Parameters of QoL as assessed by ESAS-r demonstrated a statistically significant change $(\mathrm{p}<0.05)$ in anxiety, physical function and emotional function. All other parameters as listed in Table 3 were not found to have a statistically significant change over treatment course from baseline to post-treatment four. Mean anxiety decreased from 3.15 to 2.35 ( $\mathrm{p}=0.05)$, physical function from 11.91 to $9.41(\mathrm{p}=0.04)$ and emotional function from 5.03 to 3.82. As a single measure score anxiety did not meet the MCID of a decrease of one point, emotional function did not meet the MCID of a decrease of two points and physical function did not meet the MCID of a decrease by three points. As such, only overall total symptom distress score was found to be both statistically and clinically significant.

\section{Discussion}

The mean global health status score for this patient cohort with advanced GEP-NETs using EORTC QLQ-C30 with GI.NET 21 was 67.63 at baseline. In comparison, the mean global health status for all cancer patients with advanced cancer cited in the literature is 61.5 [41]. The mean total symptom distress score for the study cohort as measured by ESAS-r was 19.85. In the literature, the mean total symptom distress score for all cancer patients, 


\begin{tabular}{|c|c|c|}
\hline \multirow[t]{2}{*}{ ESAS-r } & \multicolumn{2}{|c|}{ Mean (SD) } \\
\hline & Baseline & Post-treatment 4 \\
\hline Tiredness & $\begin{array}{l}3.47 \\
(3.10)\end{array}$ & $\begin{array}{l}2.68 \\
(2.41)\end{array}$ \\
\hline Nausea & $\begin{array}{l}0.97 \\
(1.67)\end{array}$ & $\begin{array}{l}0.74 \\
(1.29)\end{array}$ \\
\hline Lack of appetite & $\begin{array}{l}1.47 \\
(2.26)\end{array}$ & $\begin{array}{l}1.00 \\
(1.60)\end{array}$ \\
\hline Dyspnea & $\begin{array}{l}2.03 \\
(2.77)\end{array}$ & $\begin{array}{l}2.09 \\
(2.27)\end{array}$ \\
\hline Overall well being & $\begin{array}{l}2.94 \\
(2.69)\end{array}$ & $\begin{array}{l}2.21 \\
(2.32)\end{array}$ \\
\hline Physical score & $\begin{array}{l}11.91 \\
(11.89)\end{array}$ & $\begin{array}{l}9.41^{*} \\
(9.68)\end{array}$ \\
\hline Emotional score & $\begin{array}{l}5.03 \\
(5.51)\end{array}$ & $\begin{array}{l}3.82^{*} \\
(4.63)\end{array}$ \\
\hline Total symptom distress score & $\begin{array}{l}19.85 \\
(18.37)\end{array}$ & $\begin{array}{l}15.38^{*, * *} \\
(15.21)\end{array}$ \\
\hline
\end{tabular}

with advanced cancer, it is approximately 38.6 [42]. As such, baseline scores reflective of overall QoL as calculated by both the EORTC and ESAS0-r were similar in that both scores indicated overall relatively good baseline QoL in patients with SB-NETs. This relatively higher QoL in patients with advanced SB-NETs in comparison with patients with advanced cancer of other origins is supported in the literature [43].

With regard to individual symptom scores, the ESAS-r detected three statistically significant individual parameter changes in anxiety, physical and emotional function. These improvements postinduction treatment were not, however, clinically significant. The EORTC detected four statistically significant parameter changes in endocrine symptoms, GI symptoms, diarrhea and insomnia. These detected improvements postinduction therapy were all clinically significant as change in score met the established MCID. No other studies are available for comparison with regards to evaluation of ESAS-r in NET patients. However, the results of this EORTC analysis are consistent with the results of the few other available studies evaluating QoL, especially those of patients with progressive NETs in which Lutetium therapy has been associated with a statistically and clinically significant improvements in QoL parameters [22-26,44,45].

The EORTC QLQ-C30, due to the addition of the GI.NET 21 was better able to capture symptomology with regards to specific symptoms and tumor specific features such as diarrhea and endocrine and GI symptoms in comparison with the ESAS-r. These endocrine-specific symptoms proportionally provide a large amount of the symptom burden and are important to assess in this population. The ability to individually assess these parameters may provide greater insight into potential symptoms to target preferentially in treatment and thereby improve QoL.

Conversely, the ESAS-r was not able to detect significant changes in individual symptom parameters, however it did detect an improvement in overall QoL. This is likely a result of the lack of NET-specific symptoms directly assessed by the ESAS-r. Consequently, the improvement in individual domains such as endocrine and GI symptoms were simply reflected as overall improved QoL. Thus, the ESAS-r although not as specific the ESAS-r does detect changes in overall QoL. This aspect may not be as useful in identifying symptoms to target but can help clinicians and patients track overall progress during treatment. 
We must also consider that in order for QoL assessment to have a clinical uptake the tool must be easy to score and interpret by the clinician. In this regard, the scoring system of the ESAS-r allows for easier clinician interpretation [46]. Additionally, the simple inclusion of this assessment may prompt patient-physician discussion and improve care [9]. However, ideally the QoL tool used would accurately identify problems such that they can then be identified and addressed in treatment decisions [46]. The EORTC QLQ-C30 with GI NET.21 serves this function but is more complex and cumbersome to use. Its clinical use could be facilitated by computerized assessment which would allow for quick assessment and scoring and a system which can easily track patient changes over time with serial assessment [47]. Alternatively, physicians could potentially consider use of the ESAS- $\mathrm{r}$ as a more general tracking tool and subsequently employ the EORTC questionnaire when deterioration in overall QoL is identified to help identify exact symptomology and then adequacy of specific treatment.

Limitations of this study are largely in relation to the study design and assessment tools. As this was a substudy within a clinical trial, the population included was restricted based on the study inclusion and exclusion criteria. Most notably patients at extremens of age and with poor ECOG $($ ECOG $>2$ ) were not enrolled and thus not included for analysis and our results may not be generalizable to these populations who have been demonstrated to have more extremes of QoL. With regards to QoL assessment limitation is restricted with regards to participation bias as assessment is performed by patient-reported parameter and this potential bias, while it cannot be directly avoided in QoL assessment, should be considered in the interpretation of these results. At last, with the assessment of QoL, global measures could be influenced by factors other than treatment response such as anxiety at the initiation of new treatment, thereby biasing results toward a benefit as patient anxiety could decrease as they become accustomed to treatment. As we did not find comparable change in the global scales between the EORTC and ESAS assessments, however this contribution is thought to be minimal.

\section{Conclusion}

Assessing QoL in clinical practice allows for the effective evaluation and treatment of key patient concerns and symptoms, which is especially relevant for patients with advanced cancer where treatment is not typically provided with curative intent. Each QoL assessment tool has its inherent benefits and limitations - all of which must be considered in clinical use. ESAS-r provides a quick, simple and easy to interpret tool for QoL evaluation and can allow physicians to follow general trends in their patient's QoL.

The EORTC QLQ-C30 with GI NET.21 is NET specific and validated tool demonstrated to detect changes in QoL in patients with NETs. The EORTC tool is more complex and time consuming but can be incorporated into clinical practice if simple measures such as computerized assessment and scoring are established. The benefit of this tool is that it can help identify problem areas that need to be better addressed.

Regardless, inclusion of any QoL assessment tool is preferential as it can prompt discussion and facilitate better patient care. Our results suggest that the optimal assessment tool is the EORTC QLQ-C30 with GI NET.21 and as such this is the QoL assessment tool we recommend if institutional resources allow. If however, use of the EORTC QLQ-C30 with GI NET.21 is not feasible for all patients, the ESAS-r can potentially be used as a screening tool for identification of patients with low QoL who can then be further evaluated with the EORTC assessment tool.

\section{Summary points}

- Lu-DOTA-TATE treatment of progressive metastatic small bowel is associated with statistically and clinically significant improvement in QoL.

- NET-specific QoL assessment tools such as the European Organization for Research and Treatment of Cancer QLQ-C30 GI.NET 21 allow for a more specific assessment of QoL parameters in these patients.

- General QoL assessment tools such as the ESAS-r still detect changes in overall QoL in these patients and can be more easily adopted into clinical practice. 
Financial \& competing interests disclosure

Funding for this Phase II trial was provided in part by grants from the Alberta Heritage Foundation for Medical Research, Canadian Institute of Health Research and Alberta Cancer Foundation. No funding agencies had a role in study design, interpretation or reporting. The authors have no other relevant affiliations or financial involvement with any organization or entity with a financial interest in or financial conflict with the subject matter or materials discussed in the manuscript apart from those disclosed.

No writing assistance was utilized in the production of this manuscript.

Ethical conduct of research

Ethics approval was obtained from both the University of Alberta research ethics board and from Health Canada. Accordingly, written informed consent was obtained and maintained from all subjects. Clinical Trial Number: NCT01876771 The authors state that they have obtained appropriate institutional review board approval or have followed the principles outlined in the Declaration of Helsinki for all human or animal experimental investigations. In addition, for investigations involving human subjects, informed consent has been obtained from the participants involved.

\section{Open access}

This work is licensed under the Attribution-NonCommercial-NoDerivatives 4.0 Unported License. To view a copy of this license, visit http://creativecommons.org/licenses/by-nc-nd/4.0/

\section{References}

Papers of special note have been highlighted as: $\bullet \bullet$ of considerable interest

1. Tinetti ME, Naik AD, Dodson JA. Moving from disease-centered to patient goals - directed care for patients with multiple chronic conditions. JAMA Cardiol. 1, 9-10 (2016).

2. Bardes CL. Defining 'patient-centered medicine'. N. Engl. J. Med. 366(9), 782-783 (2012).

3. Basch E. Patient-reported outcomes - harnessing patients' voices to improve clinical care. N. Engl. J. Med. 376(2), 105-108 (2017).

4. Leppert W, Gottwald L, Forycka M. Clinical practice recommendations for quality of life assessment in patients with gynecological cancer. Menopausal Rev. 4(4), 271-282 (2015).

5. Homsi J, Walsh D, Rivera $\mathrm{N}$ et al. Symptom evaluation in palliative medicine: patient report vs systematic assessment. Support Care Cancer 14(5), 444-453 (2006).

6. Johnstone PAS, Lee J, Zhou J-M et al. A modified Edmonton symptom assessment scale for symptom clusters in radiation oncology patients. Cancer Med. 6(9), 2034-2041 (2017).

7. Dobrozsi S, Panepinto J. Patient-reported outcomes in clinical practice. Hematology Am. Soc. Hematol. Educ. Program. 2015(1), 501-506 (2015).

8. Jacobsen PB, Davis K, Cella D. Assessing quality of life in research and clinical practice. Oncology (Williston Park). 16(9 Suppl. 10), 133-139 (2002).

9. King S, Exley J, Parks $S$ et al. The use and impact of quality of life assessment tools in clinical care settings for cancer patients, with a particular emphasis on brain cancer: insights from a systematic review and stakeholder consultations. Qual. Life Res. 25(9), 2245-2256 (2016).

10. Moher D, Shamseer L, Clarke M et al. Preferred reporting items for systematic review and meta-analysis protocols (PRISMA-P) 2015 statement. Syst. Rev. 4, 1 (2015).

11. Nardi EA, McCanney J, Winckworth-Prejsnar K et al. Redefining quality measurement in cancer care. J. Natl Compr. Canc. Netw. 16(5), 473-478 (2018).

12. Dasari A, Shen C, Halperin D et al. Trends in the incidence, prevalence, and survival outcomes in patients with neuroendocrine tumors in the United States. JAMA Oncol. 26(13), 2124-2130 (2017).

13. Yalcin S, Bayram F, Erdamar S, Kucuk O, Oruc N, Coker A. Guidelines/recommendations gastroenteropancreatic neuroendocrine tumors: recommendations of Turkish multidisciplinary neuroendocrine tumor study group on diagnosis, treatment and follow-up. 13(2), 271-282 (2017).

14. Janson ET, Sorbye H, Welin S et al. Nordic guidelines 2014 for diagnosis and treatment of gastroenteropancreatic neuroendocrine neoplasms. Acta Oncol. 53(10), 1284-1297 (2014).

15. Howe JR, Cardona K, Fraker DL et al. The surgical management of small bowel neuroendocrine tumors. Pancreas 46(6), 715-731 (2017).

16. Garcia-Carbonero R. Gastroenteropancreatic neuroendocrine tumors: current perspectives and future challenges. Cancer Metastasis Rev. 33(1), 343-4 (2013).

17. Trofimiuk-Muldner M, Lewkowicz E, Wysocka K et al. Epidemiology of gastroenteropancreatic neuroendocrine neoplasms in Krakow and Krakow district in 2007-2011. Endokrynol. Pol. 68(1), 42-46 (2017). 
18. Mosquera C, Koutlas NJ, Fitzgerald TL. Localized high-grade gastroenteropancreatic neuroendocrine tumors: Defining prognostic and therapeutic factors for a disease of increasing clinical significance. Eur. J. Surg. Oncol. 42(10), 1471-1477 (2016).

19. Neychev V, Kebebew E. Management options for advanced low or intermediate grade gastroenteropancreatic neuroendocrine tumors: review of recent literature. Int. J. Surg. Oncol. 2017, 6424812 (2017).

20. Kwekkeboom DJ, Krenning EP, Lebtahi R et al. ENETS consensus guidelines for the standards of care in neuroendocrine tumors: Peptide receptor radionuclide therapy with radiolabeled somatostatin analogs. Neuroendocrinology 90(2), 220-226 (2009).

21. Kocha W, Maroun J, Kennecke $\mathrm{H}$ et al. Consensus recommendations for the diagnosis and management of well-differentiated gastroenterohepatic neuroendocrine tumours: a revised statement from a Canadian National Expert Group. Curr. Oncol. 17(3), 49-64 (2010).

22. Marinova M, Mücke M, Mahlberg L et al. Improving quality of life in patients with pancreatic neuroendocrine tumor following peptide receptor radionuclide therapy assessed by EORTC QLQ-C30. Eur. J. Nucl. Med. Mol. Imaging. 45(1), 38-46 (2017).

23. Teunissen JJM, Kwekkeboom DJ, Krenning EP. Quality of life in patients with gastroenteropancreatic tumors treated with 177Lu-DOTA0,Tyr3] octreotate. J. Clin. Oncol. 22(13), 2724-2729 (2004).

24. Marinova M, Muecke M, Fischer F et al. Peptide receptor radionuclide therapy (PRRT) of midgut neuroendocrine tumors: assessment of quality of life. J. Nucl. Med. 57(Suppl. 2), 1437-1437 (2016).

25. Khan S, Krenning EP, van Essen M, Kam BL, Teunissen JJ, Kwekkeboom DJ. Quality of life in 265 patients with gastroenteropancreatic or bronchial neuroendocrine tumors treated with [177Lu-DOTA0,Tyr3] Octreotate. J. Nucl. Med. 52(9), 1361-1368 (2011).

26. Strosberg J, Wolin E, Chasen B et al. Health-related quality of life in patients with progressive midgut neuroendocrine tumors treated with 177Lu-dotatate in the Phase III NETTER-1 trial. J. Clin. Oncol. 36(25), 2578-2584 (2018).

-• Seminal Phase III trial assessing Lutetium treatment for patients with midgut neuroendocrine tumors. Demonstrated benefit of Lutetium in relation to treatement with high dose octreotide.

27. Rauenzahn SL, Schmidt S, Aduba IO, Jones JT, Ali N, Tenner LL. Integrating palliative care services in ambulatory oncology: an application of the edmonton symptom assessment system. J. Oncol. Pract. 13(4), e401-e407 (2017).

28. Bjordal K. Impact of quality of life measurement in daily clinical practice. Ann. Oncol. 15(Suppl. 4), 279-282 (2004).

29. Detmar SB, Muller MJ, Wever LD, Schornagel JH, Aaronson NK. The patient-physician relationship. Patient-physician communication during outpatient palliative treatment visits: an observational study. JAMA 285(10), 1351-1357 (2001).

30. Aaronson NK, Ahmedzai S, Bergman B et al. The European Organization for Research and Treatment of Cancer QLQ-C30: a quality-of-life instrument for use in international clinical trials in oncology. J. Natl Cancer Inst. 85(5), 365-376 (1993).

31. Hui D, Bruera E. The Edmonton symptom assessment system 25 years later: past, present, and future developments. J. Pain Symptom Manage. 53(3), 630-643 (2017).

32. Bruera E, Kuehn N, Miller MJ, Selmser P, Macmillan K. The Edmonton symptom assessment system (ESAS): a simple method for the assessment of palliative care patients. J. Palliat. Care 7(2), 6-9 (1991).

33. Watanabe SM, Nekolaichuk C, Beaumont C, Johnson L, Myers J, Strasser F. A multicenter study comparing two numerical versions of the Edmonton Symptom Assessment System in palliative care patients. J. Pain Symptom Manage. 41(2), 456-468 (2011).

34. Yadegarfar G, Friend L, Jones L et al. Validation of the EORTC QLQ-GINET21 questionnaire for assessing quality of life of patients with gastrointestinal neuroendocrine tumours. Br. J. Cancer. 108(2), 301-310 (2013).

35. Osoba D, Rodrigues G, Myles J, Zee B, Pater J. Interpreting the significance of changes in health-related quality-of-life scores. J. Clin. Oncol. 16(1), 139-144 (1998).

36. Bedard G, Zeng L, Zhang L et al. Minimal important differences in the EORTC QLQ-C30 in patients with advanced cancer. Asia. Pac. J. Clin. Oncol. 10(2), 109-117 (2014).

37. Maringwa J, Quinten C, King M et al. Minimal clinically meaningful differences for the EORTC QLQ-C30 and EORTC QLQ-BN20 scales in brain cancer patients. Ann. Oncol. 22(9), 2107-2112 (2011).

38. Hui D, Shamieh O, Paiva CE et al. Minimal clinically important difference in the physical, emotional and total symptom distress scores of the edmonton symptom assessment system. J. Pain Symptom Manage. 51(2), 262-269 (2016).

39. Hannon B, Dyck M, Pope A et al. Modified Edmonton symptom assessment system including constipation and sleep: Validation in outpatients with cancer. J. Pain Symptom Manage. 49(5), 945-952 (2015).

40. Hui D, Shamieh O, Paiva CE et al. Minimal clinically important differences in the Edmonton symptom assessment scale in cancer patients: a prospective multicenter study. Cancer. 121(17), 3027-3035 (2015).

41. Scott NW, Fayers PM, Aaronson NK et al. EORTC-QLQ-C30 reference values. Brussels, Belgium (2018). www.eortc.org/app/uploads/sites/2/2018/02/reference_values_manual2008.pdf

42. Cheung WY, Le LW, Gagliese L, Zimmermann C. Age and gender differences in symptom intensity and symptom clusters among patients with metastatic cancer. Support Care Cancer 19(3), 417-423 (2011).

43. Larsson G, Sjödén PO, Oberg K, Eriksson B, von Essen L. Health-related quality of life, anxiety and depression in patients with midgut carcinoid tumours. Acta Oncol. (Madr) 40(7), 825-831 (2001). 
44. Marinova M, Muecke M, Mahlberg L et al. Quality of life assessment following peptide receptor radionuclide therapy (PRRT) of pancreatic neuroendocrine tumor (P-NET). J. Nucl. Med. 57, 2016-2017 (2016).

45. Mateo-Vito I, Amlani A, Leshen R, Mcmeekin H, Davis N, Navalkissoor S. Evaluation of quality of life after each cycle in patients treated with Peptide Receptor Radionuclide Therapy (PRRT). Endocr. Abstr. 3947, 1-2 (2016).

46. Higginson IJ, Carr A. Using quality of life measures in the clinical setting. BMJ 322, 1297-1300 (2001).

47. Wintner LM, Sztankay M, Aaronson N et al. The use of EORTC measures in daily clinical practice - a synopsis of a newly developed manual. Eur. J. Cancer. 68, 73-81 (2016). 